\title{
Effort-Based Reinforcement Processing and Functional Connectivity Underlying Amotivation in Medicated Patients with Depression and Schizophrenia
}

\author{
IIl Ho Park, ${ }^{1,2 *}$ Boung Chul Lee, ${ }^{3 *}$ Jae-Jin Kim, ${ }^{4}$ Joong Il Kim, ${ }^{2}$ and Min-Seung Koo ${ }^{1}$ \\ ${ }^{1}$ Department of Psychiatry and Behavioral Neurosciences, and 'Institute for Integrative Medicine, Catholic Kwandong University, International St. Mary's \\ Hospital, Incheon 22711, Republic of Korea, ${ }^{3}$ Department of Psychiatry, Hallym University Hangang Sacred Heart Hospital, Seoul 07247, Republic of Korea, \\ and ${ }^{4}$ Department of Psychiatry, Yonsei University College of Medicine, Gangnam Severance Hospital, Seoul 06273, Republic of Korea
}

\begin{abstract}
Amotivation is a common phenotype of major depressive disorder and schizophrenia, which are clinically distinct disorders. Effective treatment targets and strategies can be discovered by examining the dopaminergic reward network function underlying amotivation between these disorders. We conducted an fMRI study in healthy human participants and medicated patients with depression and schizophrenia using an effort-based reinforcement task. We examined regional activations related to reward type (positive and negative reinforcement), effort level, and their composite value, as well as resting-state functional connectivities within the meso-striatalprefrontal pathway. We found that integrated reward and effort values of low effort-positive reinforcement and high effort-negative reinforcement were behaviorally anticipated and represented in the putamen and medial orbitofrontal cortex activities. Patients with schizophrenia and depression did not show anticipation-related and work-related reaction time reductions, respectively. Greater amotivation severity correlated with smaller work-related putamen activity changes according to reward type in schizophrenia and effort level in depression. Patients with schizophrenia showed feedback-related putamen hyperactivity of low effort compared with healthy controls and depressed patients. The strength of medial orbitofrontal-striatal functional connectivity predicted work-related reaction time reduction of high effort negative reinforcement in healthy controls and amotivation severity in both patients with schizophrenia and those with depression. Patients with depression showed deficient medial orbitofrontal-striatal functional connectivity compared with healthy controls and patients with schizophrenia. These results indicate that amotivation in depression and schizophrenia involves different pathophysiology in the prefrontal-striatal circuitry.
\end{abstract}

Key words: depression; functional neuroimaging; motivation; schizophrenia; ventral tegmental area

\section{Significance Statement}

Amotivation is present in both depression and schizophrenia. However, treatment involves the use of drugs that enhance serotonin activity in depression and inhibit serotonin and dopamine activity in schizophrenia. Understanding how motivation processed in the mesocorticolimbic and nigostriatal pathways is affected in depression and schizophrenia is important in discovering treatment targets and strategies for amotivation. To our knowledge, this is the first study to compare patients with depression and schizophrenia in a common functional construct. By using an effort-based reinforcement task and examining resting-state functional connectivity in the dopaminergic network, we propose that difference in striato- orbitofrontal dysfunction in effort-based reinforcement between depression and schizophrenia may be related to differences in the extent of functional dysconnectivity in the dopaminergic pathway.

\section{Introduction}

Anhedonia and avolition, attributed to deficit in the reward pathway, are common phenotypes of schizophrenia and major de-

\footnotetext{
Received Aug. 9, 2016; revised Feb. 25, 2017; accepted Feb. $28,2017$.

Author conftributions: I.H.P. and J.-J.K. designed research; I.H.P., B.C.L., and M.-S.K. performed research; I.H.P. and J.I.K. analyzed data; I.H.P., B.C.L., and J.-J.K. wrote the paper.

This work was supported by the Basic Science Research Program through the National Research Foundation of Korea funded by the Korean government (Ministry of Education, Culture, Sports, Science and Technology Grant NRF-2012R1A1A1010199). We thank Yongtak Jin and Deokman Kim for help with clinical data collection, Ju-Yeol
}

pressive disorder. In schizophrenia, anhedonia and avolition are difficult to treat and persist longer than psychotic symptoms, whereas, in depression, anhedonia and avolition are more likely to recover with treatment. Moreover, serotonin-dopamine receptor antagonists and serotonin reuptake inhibitors are used

\footnotetext{
Lee for data collection and analysis, Hyunwoong Ko for data analysis, Jisoo Kim and Hanna Huh for data collection, and Eunjung Choi for programming the experimental paradigm.

The authors declare no competing financial interests.

*I.H.P. and B.C.L. contributed equally to this work.
} 
mainly for treating schizophrenia and depression, respectively. Understanding the difference in the underlying neural functioning of amotivation in schizophrenia and depression may help in discovering effective treatment targets for negative symptoms in schizophrenia.

The effort-based decision-making paradigm is a translational model that can be applied to motivational anhedonia in major depressive disorder and to negative symptoms in schizophrenia (Treadway et al., 2012a; Gold et al., 2013). According to this model, amotivation is viewed as a dysfunctional decision-making process of either overestimation of cost for obtaining reward or failure to integrate optimally cost/benefit information that results in disengaging approach behavior. This paradigm involves regions comprising the dopaminergic reward pathway: the anterior cingulate cortex in evaluation and execution of cost-effective options, the nucleus accumbens (NAc) in the anticipation and drive to attain a preferred option, the putamen in computations for choices involving effort costs, and the orbitofrontal cortex (OFC) in encoding and integrating the relative values of outcomes (Assadi et al., 2009; Kurniawan et al., 2010; Lee et al., 2015).

Reward processing in the prefrontal-striatal circuitry has been studied extensively using monetary incentive paradigms and its dysfunction has been observed in patients with depression or schizophrenia. Altered associations of anterior cingulate cortex activation with anticipation of gains instead of losses and deficient activations of the NAc and caudate nucleus in response to receipt of monetary gains have been observed in depressed individuals (Knutson et al., 2008; Pizzagalli et al., 2009). In a study of schizophrenia, activations in the medial and lateral prefrontal cortex, lateral temporal cortex, and amygdala were attenuated in response to outcomes of greater gains than losses (Waltz et al., 2010). Other studies have suggested that reward anticipationrelated and reward receipt-related ventral striatum functioning and reward engagement-related anterior cingulate cortex functioning, respectively, underlie the development of apathy, depressive symptoms, and social amotivation in schizophrenia (Simon et al., 2010; Park et al., 2015). A few studies have focused on the ventral tegmental area in psychotic patients. These studies found that ventral tegmental area activity was attenuated during anticipation and was abnormal in response to reward prediction error (Murray et al., 2008; Nielsen et al., 2012). These studies suggest that neural processing in the prefrontal-striatal circuitry is dysfunctional in patients with depression and those with schizophrenia. However, whether there are differences in the functioning of the dopaminergic reward pathway between depression and schizophrenia is largely unknown.

In this study, we examined the meso/nigro-striatal-prefrontal circuitry function associated with effort-based reinforcement processing in medicated patients with schizophrenia or major depressive disorder. We hypothesized that amotivation in depression and schizophrenia is associated with different patterns of disruption in effort-based reinforcement activity and functional connectivity within the meso/nigral-striatal-orbitofrontal circuitry.

This research was presented as a poster at the 5th Biennial Schizophrenia International Research Society Conference in Florence, Italy, on April 2-6, 2016.

Correspondence should be addressed to $1 \mathrm{H}$ Ho Park, M.D., Ph.D., Department of Psychiatry and Behavioral Neurosciences, Institute of Integrative Medicine, Catholic Kwandong University International St. Mary's Hospital, 25 , Simgok-ro 100beon-gil, Seo-gu, Incheon, 22711 South Korea. E-mail: eihpark@gmail.com.

DOI:10.1523/JNEUROSCI.2524-16.2017

Copyright $\odot 2017$ the authors $\quad 0270-6474 / 17 / 374371-11 \$ 15.00 / 0$

\section{Materials and Methods}

Participants. Twenty-two outpatients with depression, 23 outpatients with schizophrenia, and 31 healthy individuals of either sex matched by age and sex participated in this study. All participants consented to the procedures approved by the Institutional Review Board of Hangang Sacred Heart Hospital. Patients met DSM-IV-TR criteria for major depressive disorder or schizophrenia and were on antipsychotics and/or antidepressants. Patients with any past or present medical or neurological illnesses, comorbid psychiatric disorders, substance use disorder, or mental retardation were excluded. All participants completed the 17item Beck Depression Inventory (BDI) (Beck et al., 1961) and Apathy Evaluation Scale (AES) (Marin et al., 1991; Lee et al., 2013) and schizophrenia or depression patients were rated with the 17-item Hamilton Depression Rating Scale (HDRS) (Hamilton, 1960) and the Scale for the Assessment of Negative Symptoms (SANS) (Andreasen, 1984a) by a psychiatrist. In addition, the Scale for the Assessment of Positive Symptoms (SAPS) (Andreasen, 1984b) was administered to schizophrenia patients. The AES scores were used as subjective measure of amotivation and sum of the SANS Avolition-Apathy and Anhedonia-Associality item scores was used as an objective measure of amotivation dimension of negative symptoms (Foussias and Remington, 2010). Eight participants were excluded from data analyses due to incidental diagnosis of brain tumor, history of substance use, or omission of behavioral response, leaving 30 healthy individuals, 19 schizophrenic, and 19 depressed patients to be included in the analyses. Patients with depression and schizophrenia were less educated than healthy controls (Table 1).

Image data acquisition. Functional and structural magnetic resonance imaging (MRI) was conducted using a $1.5 \mathrm{~T}$ Siemens Magnetom Sonata Syngo MR A30. We used T2*-weighted gradient echo echoplanar imaging sequence [ 38 slices of $2.5 \mathrm{~mm}$ thickness and no gaps, repetition time $(\mathrm{TR})=3000 \mathrm{~ms}$, echo time $(\mathrm{TE})=30 \mathrm{~ms}$, flip angle $(\mathrm{FA})=90^{\circ}$, and field of view $=210 \mathrm{~mm}$ ] with a resolution of $3.3 \mathrm{~mm} \times 3.3 \mathrm{~mm} \times 2.5 \mathrm{~mm}$ to obtain blood oxygen level-dependent (BOLD) images for task activation and resting-state scans. To minimize signal loss in the OFC, we scanned imaging slices at an angle of $15^{\circ}$ from the anterior commissure-posterior commissure line (Deichmann et al., 2003). To maintain high spatial resolution, we limited the range of imaging acquisition below the superior parietal area. We obtained structural images with a resolution of 1.2 $\mathrm{mm} \times 0.8 \mathrm{~mm} \times 1.3 \mathrm{~mm}$ using a 3D T1-weighted gradient echo sequence $\left(128\right.$ slices, $\left.\mathrm{TR}=1900 \mathrm{~ms}, \mathrm{TE}=3.93 \mathrm{~ms}, \mathrm{FA}=15^{\circ}\right)$.

Effort-based reinforcement task and fMRI scan procedure. The effortbased cost-benefit valuation task developed by Croxson et al. (2009) was modified to examine the effects of reward type (i.e., positive and negative reinforcer) instead of reward magnitude on effort-based reinforcement. We were interested in the effects of reward type because prior studies suggested that negative reinforcement learning was intact in schizophrenia but aberrant in depression due to either hypersensitivity or blunted response to negative feedback (Waltz et al., 2007; Eshel et al., 2010; Strauss et al., 2011). A visual cue indicating the trial condition was presented for 1.5-3.0 s, followed by a signal to press the start button within $2 \mathrm{~s}$. After a brief delay of $0.5 \mathrm{~s}$, two switched-off light bulbs were displayed for $1 \mathrm{~s}$ and then randomly switched on and off repeatedly 3,4 , 15, or 20 times. Participants were instructed to switch off the light bulb as quickly as possible by pressing a right or left button. After a $2 \mathrm{~s}$ delay, they received one of the following feedbacks: a gain of 500 South Korean won (\$0.45), no gain, a loss, or no loss. The feedback also included the accumulated amount of money and was displayed for $2 \mathrm{~s}$. The task was conducted in 3 runs, each consisting of 40 trials in a random sequence. The task trials were composed of four reinforcement and one null condition according to reward type (i.e., monetary gain vs loss avoidance) and effort level (i.e., 3/4 vs 15/20 button presses). These led to positive reward with low or high effort (PL or $\mathrm{PH}$ ), negative reward with low or high effort (NL or $\mathrm{NH}$ ), and no reward with no effort (i.e., one button press without reward; NN) (Fig. 1).

All participants performed a practice task before MRI scanning. After structural image acquisition, we conducted fMRI scanning of three task runs and two resting-state runs, in which the first and fourth runs were resting-state runs. During a resting-state run, participants were in- 
Table 1. Demographic and clinical data

\begin{tabular}{|c|c|c|c|c|}
\hline & Control $(n=30)$ & Depression $(n=19)$ & Schizophrenia $(n=19)$ & $F / \chi^{2} / t(p)$ \\
\hline Age & $35.4(10.1)$ & $37.2(11.4)$ & $36.6(7.3)$ & $0.22(0.81)$ \\
\hline Sex (female/male) & $17 / 13$ & $12 / 7$ & $10 / 9$ & $0.44(0.80)$ \\
\hline Years of education & $15.7(2.4)$ & $13.7(4.2)$ & $12.8(2.0)$ & $\begin{array}{r}6.52(0.003) \\
C>S, C>D\end{array}$ \\
\hline Duration of illness (years) & & $3.7(2.7)$ & $10.7(6.9)$ & $-4.15(<0.001)$ \\
\hline Antidepressant & & $\begin{array}{c}\mathrm{FLX}(6), \operatorname{ESC}(7), \mathrm{VNL}(1), \operatorname{PRX}+\operatorname{MRT}(1), \\
\operatorname{ESC}+\operatorname{DLX}(2), \mathrm{ESC}+\mathrm{FLX}(2)\end{array}$ & SRT (1), TRZ (1), DLX (1) & \\
\hline FLX equivalent dose & & $40.8(15.8)$ & $14.7(9.5)$ & \\
\hline Antipsychotic & & $\operatorname{QTP}(2), \operatorname{ARP}(2)$ & $\begin{array}{l}\operatorname{ARP}(3), \operatorname{RSP}(3), \operatorname{PLP}(2), \\
\operatorname{PMZ}(1), \mathrm{ZPR}(1), 0 L N(1), \mathrm{CLZ}(3), \\
\operatorname{AMS}(1), 0 L Z+\operatorname{RSP}(1), \mathrm{CLZ}+\mathrm{RSP}(1), \\
\operatorname{ARP}+\operatorname{RSP}(1), \operatorname{ARP}+\mathrm{QTP}(1)\end{array}$ & \\
\hline Chlorpromazine equivalent dose & & $122.6(185.6)$ & $312.4(250.5)$ & \\
\hline Hamilton Depression Rating Scale & & $15.5(4.2)$ & $5.7(5.0)$ & $6.54(<0.001)$ \\
\hline $\mathrm{BDI}$ & $5.4(4.0)$ & $25.9(9.7)$ & $13.4(12.7)$ & $\begin{array}{c}31.5(<0.001) \\
C<D, C<S, D>S\end{array}$ \\
\hline Apathy Evaluation Scale & $35.5(4.4)$ & $43.4(6.9)$ & $40.9(7.6)$ & $\begin{array}{l}10.53(<0.0001) \\
C<D, C<S\end{array}$ \\
\hline SANS total & & $22.3(10.6)$ & $22.05(14.27)$ & $0.05(0.96)$ \\
\hline Avolition (SANS N3 + N4) & & $14.78(6.40)$ & $11.63(8.78)$ & $1.24(0.22)$ \\
\hline SAPS total & & & $10.53(10.87)$ & \\
\hline
\end{tabular}

C, Control; D, depression; S, schizophrenia; FLX, fluoxetine; ESC, escitalopram; VNL, venlafaxine; PRX, paroxetine; MRT, mirtazapine; DLX, duloxetine; SRT, sertraline; TRZ, trazodone; QTP, quetiapine; ARP, aripiprazole; RSP, risperidone; PLP, paliperidone; PMZ, pimozide; ZPR, ziprasidone; OLN, olanzapine; CLZ, clozapine; AMS, amisulpiride.

structed to not think about anything in particular while keeping their eyes open and fixed at a circle and were scanned for $6 \mathrm{~min}$ and $24 \mathrm{~s}$. After scanning, participants chose their preferred cue on a laptop computer between two visual cue sets consisting of 60 null-versusreinforcement condition sets (i.e., absolute cue preference), 30 positive-versus-negative reward sets compared at fixed effort levels, and 30 low-versus-high effort sets of fixed reward type (i.e., relative cue preference).

Behavioral data analysis. As measures of reward anticipation and effort, reaction times of the cue response and the last switchoff response were analyzed. Mixed-model ANOVAs were conducted to examine the effects of trial condition, group and their interactions on reaction time. One-sample $t$ test of cue preference was conducted with a test value of $50 \%$.

Imaging data analysis. fMRI data were preprocessed and analyzed using SPM12 (RRID: SCR:007037). The first five volumes of each functional time series were discarded to remove non-steady-state effects caused by $\mathrm{T} 1$ saturation. All image volumes were adjusted for slice timing differences, realigned for motion correction, spatially normalized to the standard template, and then smoothed with an $8 \mathrm{~mm}$ full-width-at-half-maximum Gaussian kernel.

In the first-level analyses, design matrices were constructed by classifying events according to task phase (cue, work, or feedback), reward type (positive or negative), and effort level (low or high). The three phases were modeled by convolving cue presentation time, display time of last effort, and feedback with the canonical hemodynamic response function. Individual realignment parameters were entered as regressors to control for movement-related variance. Individual contrast maps representing reward type (i.e., $\mathrm{PH} / \mathrm{PL}, \mathrm{NH} / \mathrm{NL}$ ), effort level (i.e., $\mathrm{PH} / \mathrm{NH}$, $\mathrm{PL} / \mathrm{NL}$ ), and their composite value (i.e., $\mathrm{PL} / \mathrm{NH}, \mathrm{PH} / \mathrm{NL}$ ) were generated.

A priori region of interest (ROI) masks were generated using the PickAtlas SPM tool (http://fmri.wfubmc.edu/software/pickatlas). Regions as- sociated with effort-based decision making within the dopaminergic pathway were selected as ROIs (Assadi et al., 2009; Croxson et al., 2009). The midbrain region, including the ventral tegmental area, the substantia nigra (VTA/SN) (a sphere with a $15 \mathrm{~mm}$ radius centered at MNI coordinates of $x=0, y=-15, z=-9)$, and the NAc, including the adjacent head of the caudate nucleus ( $\mathrm{NAc} / \mathrm{CNH}$ ) (a sphere with a $10 \mathrm{~mm}$ radius at Talairach coordinates of $x= \pm 12, y=10, z=-2$ ), was defined based on prior studies (Knutson and Wimmer, 2007; Murray et al., 2008). The putamen, anterior cingulate cortex (ACC), and OFC were defined using automated anatomical labeling (Tzourio-Mazoyer et al., 2002).

Overall task activations were explored by conducting second-level voxelwise one-sample $t$ tests of contrast maps representing effort level, 


\section{Cue/Anticipation}

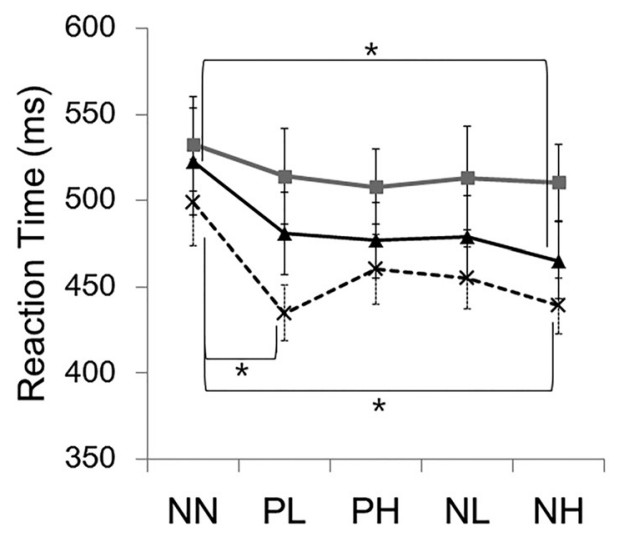

Work/Effort

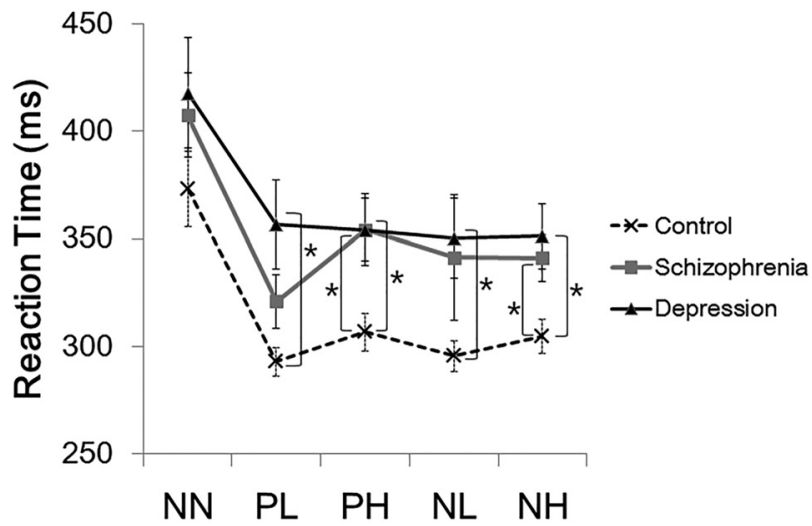

Figure 2. Mean reaction times and SEs of cue and last effort responses. * Statistically significant at Bonferroni-corrected $p<0.0125$.

reward type, and their composite value differences for each task phase in healthy controls. One-sample $t$ tests were thresholded at peak-level uncorrected $p<0.001$ with clusters consisting of 10 or more voxels.

The ROI analyses examining condition effects during the cue, work, and feedback phases were conducted separately using 3 (groups) $\times 2$ (reward types) $\times 2$ (effort levels) factorial design. Regional activities were considered statistically significant at familywise error (FWE)-corrected $p<0.05$. Post hoc $t$ tests using extracted ROI percentage signal changes were conducted at a significance level of Bonferroni-corrected $p<$ 0.0125. One-way ANOVA of effort levels, reward types, and composite values were tested for between-group differences within the ROIs at FWE-corrected $p<0.05$.

Resting-state functional connectivity analysis was performed using the CONN toolbox (http://www.nitrc.org/projects/conn, RRID: SCR: 006394). The white matter and CSF signals were regressed out and headmotion-related artifacts were reduced following a component-based noise correction method (CompCor). A temporal band-pass filter of 0.01-0.09 Hz was applied at the first-level GLM. ROI-to-ROI analyses were conducted with seed regions in the VTA/SN, putamen, $\mathrm{NAc} / \mathrm{CNH}$, and medial and lateral OFC. Between- and within-group comparisons were performed at a significance level of false discovery rate-corrected $p<0.05$.

Correlation analyses. Correlation analyses of the AES and SANS amotivation dimension scores with reward-type- and effort-level-related activity changes (i.e., positive minus negative reward and high minus low effort level, respectively) in the ROIs during the work phase and functional connectivity strength values were conducted at a significance level of Bonferroni-corrected $p<0.025$.

To confirm whether resting-state functional connectivities can mediate effort and reward-based valuation, correlations analyses of the anticipation and effort-related reaction time reductions with the resting-state functional connectivity strength values of the NAc/CHN and putamen were conducted. Anticipation and effort-related reaction time reductions were calculated by subtracting the cue and last work phase response reaction times of the reinforcement from null conditions. Among the seed regions, the NAc/CHN and putamen were selected because of their role in anticipation and instrumental action (O'Doherty et al., 2004).

\section{Results}

\section{Clinical characteristics}

Depressed patients had higher BDI and HDRS scores than schizophrenia patients. The patient groups had greater BDI and AES scores than healthy controls and did not show betweengroup differences in the SANS total or amotivation dimension score (Table 1).

\section{Reward anticipation and behavioral effort}

Absolute cue preference for positive reward was significant in healthy controls (PL 77.9\%, $t_{(29)}=6.09, p<0.001 ; \mathrm{PH} 73.6 \%$, $\left.t_{(29)}=4.65, p<0.001\right)$ and depressed patients (PL 72.4\%, $t_{(18)}=$ $3.13, p=0.006$; PH 70.2\%, $\left.t_{(18)}=3.08, p=0.006\right)$. Significant relative preferences were found only for lower effort level in healthy controls (positive reward $60.7 \%, t_{(29)}=2.79, p=0.009$; negative reward $\left.59.0 \%, t_{(29)}=3.03, p=0.005\right)$. Therefore, the effort-based reinforcement task exhibited explicit preference for positive reward and lower effort.

The mixed-model ANOVA of cue reaction time revealed a significant condition effect $\left(F_{(2.84,65)}=9.24, p<0.001\right)$ in healthy controls and depressed patients; faster PL than NN reaction was observed in healthy controls $\left(t_{(29)}=-3.75, p=0.001\right)$, and faster $\mathrm{NH}$ than $\mathrm{NN}$ reactions were found in healthy controls and schizophrenia patients $\left(t_{(29)}=-3.56, p=0.001 ; t_{(18)}=\right.$ $-3.22, p=0.005)$. Analysis of the effort reaction time showed significant condition and groups effects $\left(F_{(2.40,65)}=31.4, p<\right.$ $\left.0.001 ; F_{(2,65)}=4.80, p=0.01\right)$. Compared with healthy controls, depressed patients were slower during all four reinforcement conditions $\left(\mathrm{PL}, t_{(21.9)}=2.94, p=0.008 ; \mathrm{PH}, t_{(47)}=-2.78, p=\right.$ $0.008 ; \mathrm{NL}, t_{(23.4)}=-2.74, p=0.01 ; \mathrm{NH}, t_{(47)}=-2.94, p=$ $0.005)$, whereas schizophrenia patients were slower during $\mathrm{PH}$ and $\mathrm{NH}$ conditions $\left(t_{(47)}=-2.96, p=0.005 ; t_{(47)}=-2.70, p=\right.$ 0.01 ). No significant between-group differences in the NN cue were found (Fig. 2). No significant relationship between years of education and cue or effort reaction times were found by correlation analyses at a significance level of Bonferroni-corrected $p<0.01$ in all three groups.

\section{Effort-based reinforcement activation}

The voxelwise one-sample $t$ test results showed activity differences related to effort level, reward type, and reward-effort composite value in the areas associated with visual processing, regions in the primary and supplementary motor cortex, posterior cingulate cortex, superior and middle temporal gyrus, insular cortex, body and tail of caudate nucleus, cerebellums, inferior parietal lobule, anterior and dorsolateral prefrontal cortex, and the a priori ROIs (Fig. 3).

Cue phase factorial design ROI analyses identified VTA/SN activity changes according to effort level in healthy controls $\left(16\right.$ voxels, $F_{(1,116)}=24.5$, FWE-corrected $\left.p=0.03\right)$ and group $\times$ reward type $\times$ effort level interaction (30 voxels, $F_{(2,260)}=12.9$, FWE-corrected $\left.p=0.04\right)$. Work phase analysis revealed effort level effects in the head of the caudate nucleus (left, 47 voxels, $F_{(1,262)}=23.2$, FWE-corrected $p=0.02$; right, 38 voxels, $F_{(1,262)}=22.5$, FWE-corrected $\left.p=0.03\right)$, and reward type $\times$ effort level interaction in the putamen (34 voxels, 


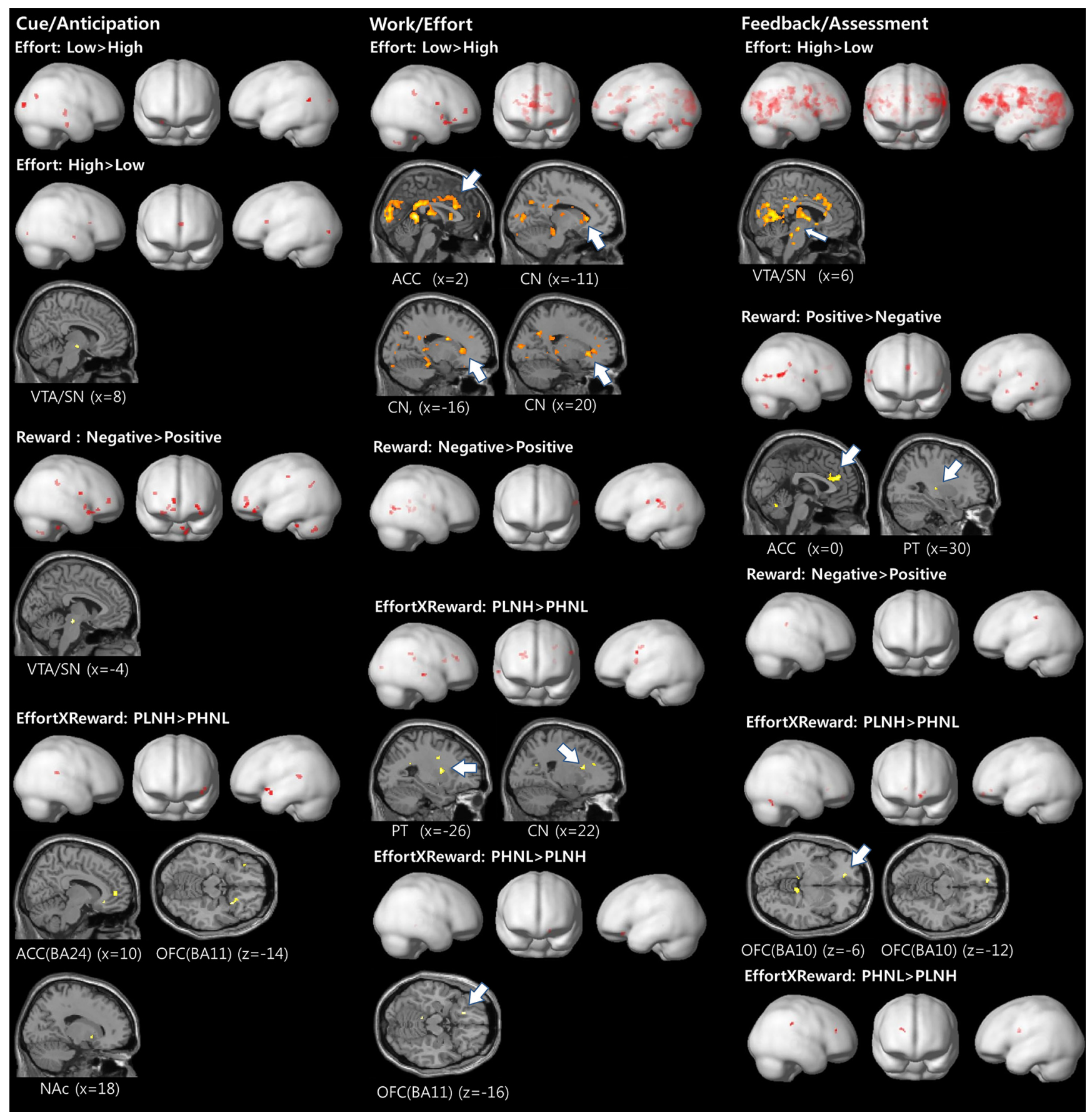

Figure 3. Voxelwise one-sample $t$ test results of task conditions in healthy controls. ROIs are shown in sections. PT, Putamen; BA, Brodmann's area; PLNH, positive reward for low effort and negative reward for high effort; PHNL, positive reward for high effort and negative reward for low effort.

$F_{(1,262)}=26.0$, FWE-corrected $\left.p=0.006\right)$. In the feedback phase analysis, the ACC (557 voxels, $F_{(1,262)}=42.9$, FWEcorrected $p<0.001$ ), head of caudate nucleus (left, 158 voxels, $F_{(1,262)}=25.1$, FWE-corrected $\left.p=0.01\right)$ and lateral OFC $(22$ voxels, $F_{(1,262)}=24.3$, FWE-corrected $\left.p=0.01\right)$ showed effort level effects, and the medial OFC (26 voxels, $F_{(1,262)}=21.4$, FWE-corrected $p<0.05)$ showed reward type $\times$ effort level interaction (Fig. 4).

In healthy controls, the right VTA/SN activity increased in response to higher effort cues of negative reward $\left(t_{(29)}=5.95\right.$, $p<0.0125)$. The head of the caudate nucleus activity was greater during lower effort work (left, $t_{(29)}=3.22, p<0.0125$; right, $\left.t_{(29)}=2.80, p<0.0125\right)$ and reward feedback for higher effort (positive reward, $t_{(29)}=2.77, p<0.0125$; negative reward, $\left.t_{(29)}=3.60, p<0.0125\right)$. The ACC and the lateral OFC showed greater activity during reward feedback for higher effort (positive reward, $t_{(29)}=3.26, p<0.0125$; negative reward, $\left.t_{(29)}=4.55, p<0.0125\right)$. During the work phase, putamen activity increased with lower effort for positive reward $\left(t_{(29)}=3.40\right.$, $p<0.0125)$ and higher effort for negative reward $\left(t_{(29)}=3.05, p<\right.$ $0.0125)$. During the feedback phase, the medial OFC activity was greater in response to positive than negative reward after low effort $\left.t_{(29)}=2.96, p<0.0125\right)$ and to negative than positive reward after high effort $\left(t_{(29)}=2.84, p<0.0125\right)$ (Fig. 4). 


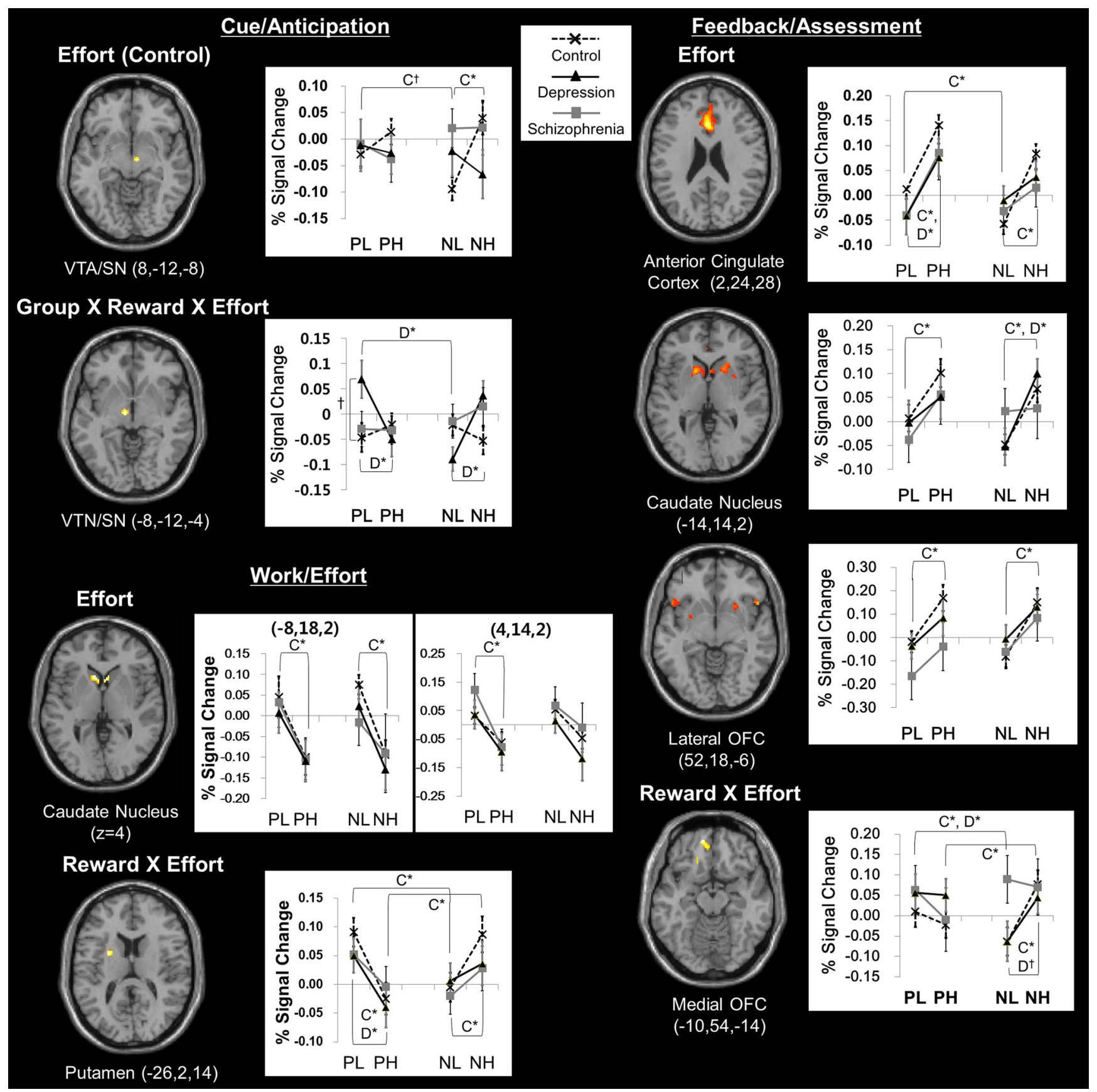

Figure 4. Brain regions showing significant activity changes related to effects of effort, reward $\times$ effort and group $\times$ reward $\times$ effort interaction and their mean percentage BOLD signal changes and SEs during the cue, work, and feedback phases. C, Control; D, depression; *Significant at Bonferroni-corrected $p<0.0125,{ }^{\dagger} p<0.025$.

In depressed patients, left VTA/SN activity was greater in response to cues representing higher effort for negative reward $\left(t_{(18)}=5.07, p<0.0125\right)$ and lower effort for positive reward $\left(t_{(18)}=2.83, p<0.0125\right)$. Moreover, activity in response to PL cue was higher in depressed patients than in healthy controls $\left(t_{(47)}=2.46, p<0.025\right)$. The putamen activity increased during lower effort work for positive reward $\left(t_{(18)}=4.91, p<\right.$ $0.0125)$. During the feedback phase, activities of the ACC and the head of the caudate nucleus increased for higher-effort positive reward and negative reward, respectively $\left(t_{(18)}=3.60\right.$, $\left.p<0.0125 ; t_{(18)}=3.93, p<0.0125\right)$. The medial OFC activity was higher for positive than negative reward feedback after low effort $\left(t_{(18)}=2.96, p<0.0125\right.$; Fig. 4). No significant relationship between years of education and ROI activities during cue, effort, or feedback were found by correlation analyses at a significance level of Bonferroni-corrected $p<0.01$ in all three groups.

Between-group differences in activities representing reward type, effort level, and composite value were found in the putamen (feedback phase, 38 voxels, $F=12.1$, cluster-level FWE-corrected $p=0.04)$. Specifically, low effort-related putamen activities during feedback phase were higher in patients with schizophrenia than healthy controls $\left(t_{(47)}=\right.$ 4.63, cluster-level FWE-corrected $p=0.01$ ) or depressed patients $\left(t_{(36)}=4.54\right.$, cluster-level FWE-corrected $\left.p=0.01\right)$ (Fig. 5). 


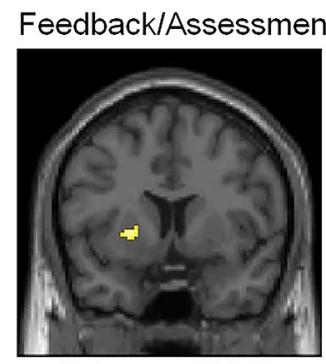

Putamen $(-24,8,0)$

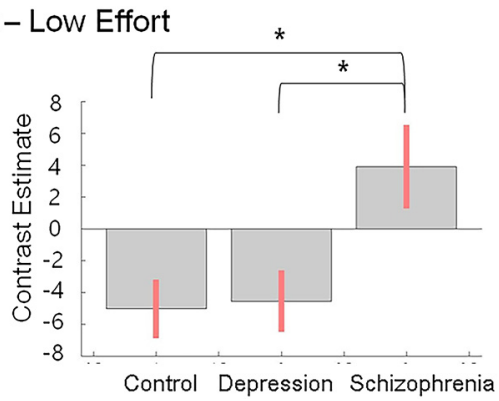

Figure 5. ROI ANOVA results of effort-related task activity during the feedback/assessment phase. Brain regions showing between-group difference and their contrast estimates and $90 \%$ confidence intervals are presented. ${ }^{*}$ Cluster-level FWE-corrected $p=0.01$.

Resting state ROI-to-ROI functional connectivity analyses In healthy controls, functional connectivity from the VTA/SN extended to all target ROIs except the lateral OFC, whereas the putamen, NAc/CNH, and the medial OFC were connected to all target ROIs. In depressed patients, no significant connectivities from the VTA/SN were observed. Moreover, connectivities from the putamen did not extend to the medial or lateral OFC and NAc/CNH connections did not extend to the VTA/SN or lateral OFC. The medial OFC showed restricted connectivities to the lateral OFC, ACC, and NAc/CNH. In schizophrenia patients, the VTA/SN-seeded connectivities were restricted to the $\mathrm{NAc} / \mathrm{CNH}$ and putamen. The putamen and NAc/CNH were significantly connected to all target ROIs and the medial OFC was significantly connected to most target ROIs except the VTA/SN (Fig. 6). Correlation analyses between years of education and functional connectivities between ROIs were found only in the VTA/SN-right NAc/CNH connection in patients with depression $(r=0.63, n=19, p=0.004)$ at a significance level of Bonferroni-corrected $p<0.005$.

No significant between-group differences in the VTA/SN and $\mathrm{NAc/CNH}$-seeded connectivities were found. However, depressed patients showed significantly less left putamen-to-right medial OFC connectivity compared with healthy controls and schizophrenia patients $\left[F_{(2,65)}=7.26\right.$, false discovery rate (FDR)- $p=001$; control, $t_{(47)}=3.21, F D R-p=0.03$; schizophrenia, $t_{(36)}=3.86, F D R-p=$ 0.004) (Fig. 6).

\section{Correlations of effort and reward-based valuation with functional connectivity}

Among cue and work phase reactions times, only reinforcement conditions with significantly faster than null conditions were used for correlation analyses with resting-state striatal functional connectivities in healthy controls. We found that the right NAc/ $\mathrm{CNH}$-right $\mathrm{ACC}$ and the right $\mathrm{NAc} / \mathrm{CNH}-\mathrm{left}$ lateral $\mathrm{OFC}$ functional connectivities positively correlated with anticipationrelated reaction time decreases in response to $\mathrm{NH}$ cues (Fig. 7A). In addition, the right putamen-left medial OFC functional connectivity negatively correlated with effort-related reaction time decreases by $\mathrm{NH}$ reinforcement (Fig. 7B).

\section{Correlations of amotivation severity with effort activity and functional connectivity}

Among work phase activities, putamen activity changes were correlated with AES and amotivation dimension scores. The AES total score was negatively correlated with low effort reward typerelated activity changes in schizophrenia patients $(r=-0.68, n=$ $19, p=0.002)$. The amotivation dimension score was negatively correlated with negative reward effort level-related activity changes in depressed patients $(r=-0.57, n=18, p=0.01)$.

AES total scores were positively correlated with right NAc/ $\mathrm{CNH}-\mathrm{left}$ medial OFC functional connectivity only in schizophrenia patients at a trend level of significance $(r=0.47, n=19$, $p=0.045$ ). The amotivation dimension score was negatively correlated with VTA/SN-left medial OFC and positively correlated with left NAc/CNH-left medial OFC functional connectivity in depressed patients. In schizophrenia patients, the amotivation dimension score was positively correlated with right NAc/CNHleft medial OFC functional connectivity (Figs. 7C,D).

The SAPS total score was positively correlated with VTA/SN-left $\mathrm{NAc/CNH}$ and negatively with right putamen-left medial OFC connectivities in patient with schizophrenia at a trend level of significance $(r=0.48, n=19, p=0.04 ; r=-0.46, n=19, p=0.047)$.

We did not find any significant correlation between antidepressant dosage and functional connectivity strength in depressed patients. However, the antipsychotic dosage was positively correlated with left NAc/CHN and bilateral ACCs connectivities (left ACC, $r=$ $0.61, n=19, p=0.005$; right ACC, $r=0.48, n=19, p=0.04)$ and with left putamen and left ACC connectivities $(r=0.56, n=19$, $p=0.01)$ in patients with schizophrenia.

\section{Discussion}

This study provides evidence that the avolition present in depression and schizophrenia could emerge from disturbances in the activation and resting-state functional connectivity in distinct parts of the meso/nigro-striatal-prefrontal circuitry associated with effort-based reinforcement. In particular, we found that amotivation severity was associated with weaker VTA/SN-medial OFC connectivity in depression and smaller effort-related putamen activity change and stronger NAc/CNH-medial OFC connectivity in both depression and schizophrenia. Depressed patients showed loss of VTA/SN connectivities, fewer striatalorbitofrontal connectivities, and deficient putamen-medial OFC connectivity, which appear to underlie the dysfunction of effortbased valuation processing. Interestingly, schizophrenia patients showed dysfunctional activation in meso/nigro-striatal-prefrontal circuitry during effort-based reinforcement despite relatively intact resting-state functional connectivity in this circuitry compared with depressed patients. Specifically, putamen was hyperactivated to low effort rewards, whereas VTA/SN resting-state connectivity to the striatum was intact and its connectivity to the prefrontal cortex was lost. Notably, patients with schizophrenia and depression were differentiated in the effort value-related putamen activity and its functional connectivity to the medial OFC. These findings suggest that examining the meso/nigro-striatalprefrontal network function in effort-based reinforcement processing can help to distinguish the underlying pathophysiology of amotivation, a common clinical phenotype, in depression and schizophrenia.

\section{Effort-based valuation and amotivation}

The patients' task performances in our study correspond to prior effort-based decision-making paradigm studies showing that patients with depression or schizophrenia are reluctant to exert effort for reward (Treadway et al., 2012a; Gold et al., 2013). We further observed differences showing that depressed patients only anticipate negative reward and are unmotivated by effortless rewards, whereas schizophrenia patients are unable to anticipate reward but are motivated by easily obtainable rewards. We identified the putamen and medial OFC as the regions associated with the integrated value of reward type and effort level during effort-based reinforcement. 


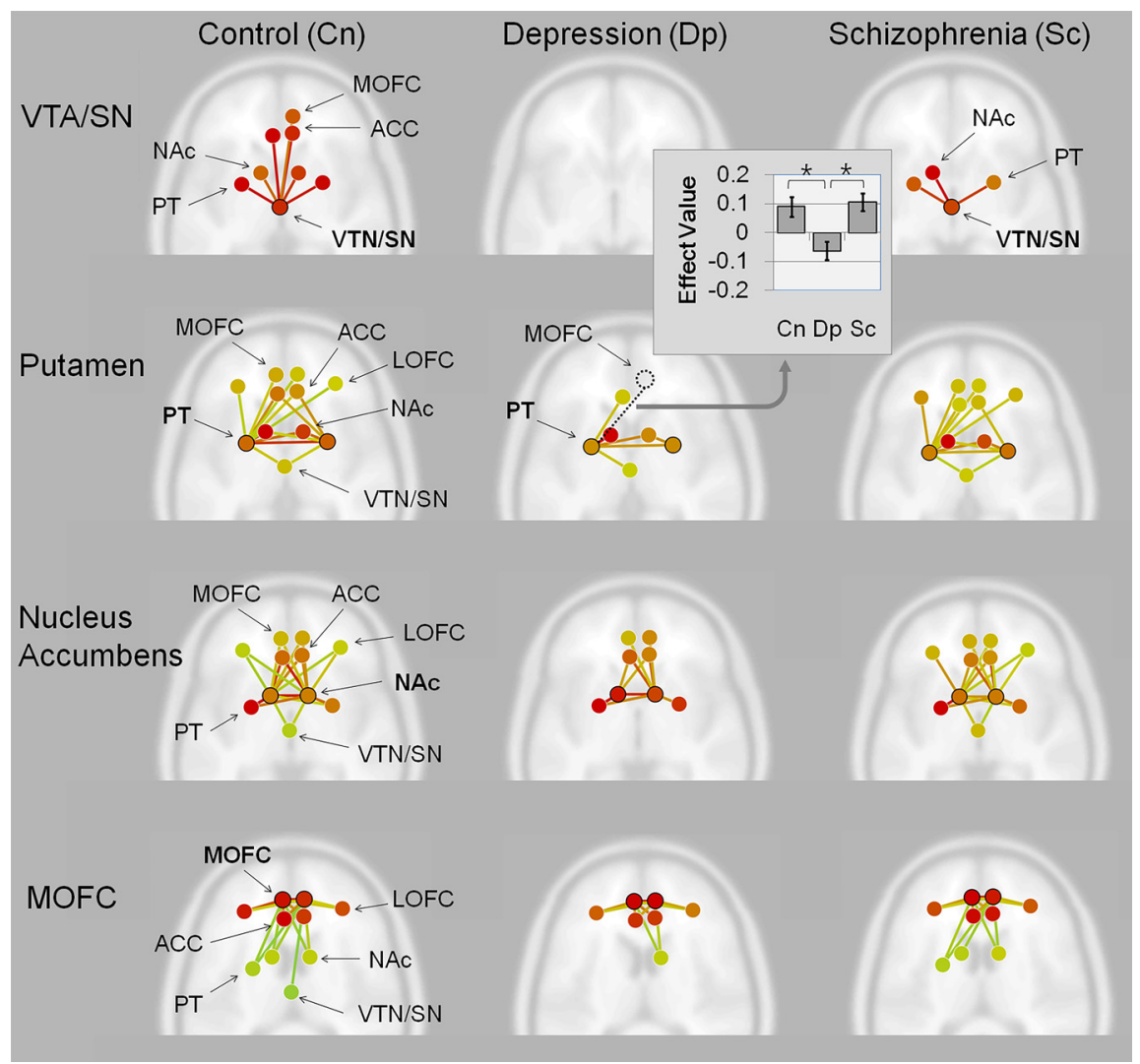

Figure 6. Within-group ROI-to-ROI resting-state functional connectivity analyses results and bar graph of between-group difference in functional connectivity. ROIs include the VTA/SN, NAc, putamen (PT), ACC, and medial and lateral orbitofrontal cortex (MOFC, LOFC). Seed regions related to effort and reward value in the VTA/SN, PT, and the MOFC and effort value related seed regions in the NAc were selected for analyses. Axial view of the brain with ROls and their functional connectivities are shown with red-to-yellow green color lines representing greater-to-less connectivity strengths at a significant FDR-corrected $p<0.05$. ${ }^{*}$ FDR- $p<0.05$.

Moreover, we found that resting-state functional connectivity of the $\mathrm{NAc/CHN}$ with ACC and lateral OFC predicted anticipatory reaction, whereas that of the putamen with medial OFC inversely predicted effort reaction to value of high effort. Notably, effort-related changes and reward-related changes in putamen activity were associated with amotivation in patients with depression and schizophrenia, respectively. Therefore, clinical avolition can be expressed by specific variation in the striatal function of reinforcement, indicating that the reward circuitry functions are altered differentially in depression and schizophrenia. In fact, we demonstrated that depressed or schizophrenia patients show loss of functional connectivities in the mesocorticolimbic and nigrostriatal pathways in different regions. In depression, the loss of functional connectivities of VTA/SN with other regions suggests that motivational drive facilitated by dopaminergic projections is insufficient. Moreover, putamen and medial OFC connectivity was deficient. In schizophrenia, connectivities of VTA/SN restricted to the $\mathrm{NAc} / \mathrm{CNH}$ and putamen suggest that dopamine-driven motivation is intact. However, loss of VTA/SN to OFC and ACC connectivities suggests otherwise.

\section{Meso-cortical pathway and amotivation}

Our observation of the associations of avolition with VTA/SN and medial OFC functional connectivity is consistent with known neuroanatomy and neurophysiology of the dopaminergic pathway. Dopaminergic projections in the mesocorticolimbic and nigrostriatal pathways function differently according to tonic or phasic dopamine release and distribution of D1 and D2 receptors in postsynaptic terminals (Dreyer et al., 2010; Grieder et al., 2012). The striatum has both D1 and D2 receptors, which facilitate and inhibit instrumental behavior through multiple relays of the GABAergic neurons forming the direct and indirect pathways (Kravitz et al., 2012; Cox et al., 2015). D1 receptors are highly distributed in the medial OFC and potentiate the prefrontal NMDA receptor-mediated response, thereby facilitating appetitive instrumental learning (Hurd et al., 2001; Baldwin et al., 2002). Therefore, our finding of loss of VTA/SN and medical OFC connectivity in schizophrenia suggests that the prepotency of positive-over-negative reinforcement value may be lost in these patients.

\section{Frontostriatal circuit and amotivation}

Our findings suggest that strengthening of medial OFC functional connectivity to the putamen suppresses effort value-related putamen activity. However, avolition was associated with stronger resting-state functional connectivity of the medial OFC to NAc instead of the putamen. These findings indicate that putamen plays a key role within the network of effort-based valuation processing in presenting the clinical phenotype of avolition.

The medial OFC has been associated with multiple roles in reward-related decision making, including reversal learning and encoding value of chosen actions (Fellows and Farah, 2003; Gläscher et al., 2009). Its function may vary depending on how and where its afferent and efferent connections operate. In relation to dopaminergic function, the striatum and ventromedial prefrontal cortex were associated with willingness to expend greater effort for larger rewards (Treadway et al., 2012b). However, the prefrontalstriatal functional connectivity represents a complex circuitry including connections with the thalamus and hippocampus. The striatum-to-prefrontal projection via the thalamus has direct reciprocal connection that can be facilitated or inhibited by either striatal D1 or D2 receptor response. Moreover, thalamic and hippocampal inputs to ventral striatum were found to be suppressed by prefrontal activation (Calhoon and O'Donnell, 2013). Overall, dysfunction in any component of the excitatory circuit may bias the frontostriatal connectivity toward inhibition of striatal activity associated with effort valuation and volition in patients with depression and schizophrenia.

Regarding the inverse relationship between the VTA/SN-medial OFC functional connectivity and avolition in the depressed patients, we propose that weakening of dopaminergic meso-prefrontal pathway may alter the function of prefrontal-striatal circuitry toward inhibiting reward drive.

\section{Pathophysiology of distal versus local circuitry}

Compared with depressed patients, schizophrenia patients showed relatively intact meso/nigral-striatum and striato-prefrontal functional connectivities, but did not show sufficient activity changes in these regions according to reward type or effort level. One possible explanation is that disturbance in the hippocampal connection to the meso/nigro-striato-prefrontal circuitry may affect reward processing (Grace et al., 2007; Lee et al., 2012). Another is that distur- 


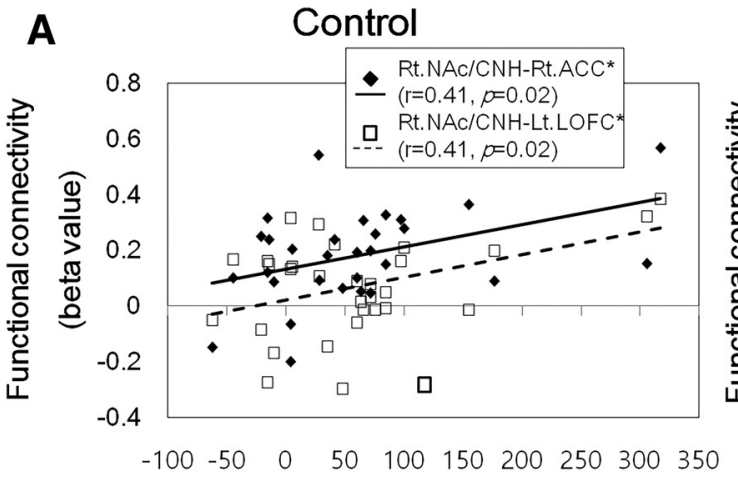

$\triangle \mathrm{NN}-\mathrm{NH}$ anticipation-related

reaction time (ms)

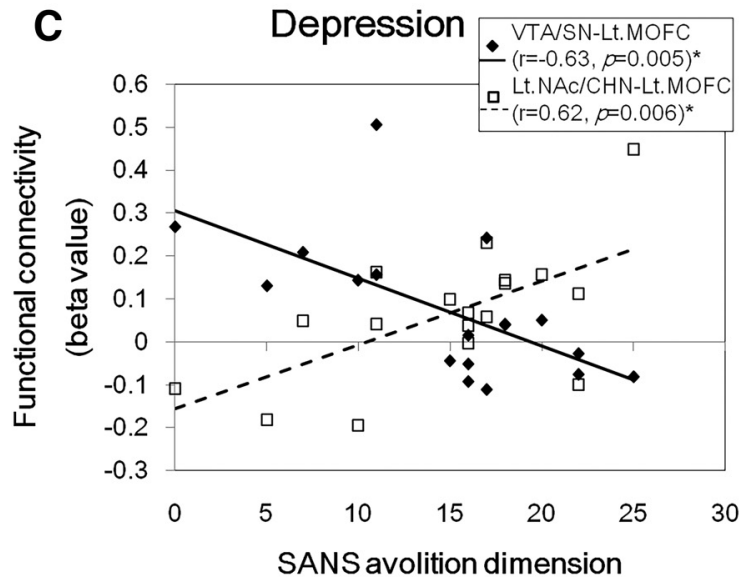

B Control

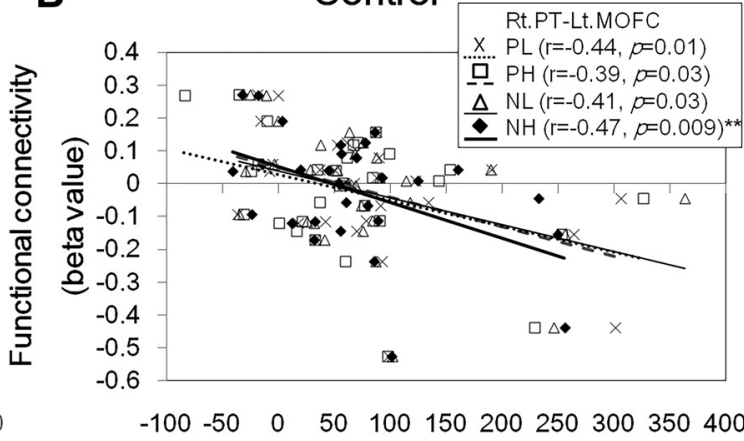

$\Delta$ Null-reinforcement effort-related reaction time ( $\mathrm{ms})$

D Schizophrenia

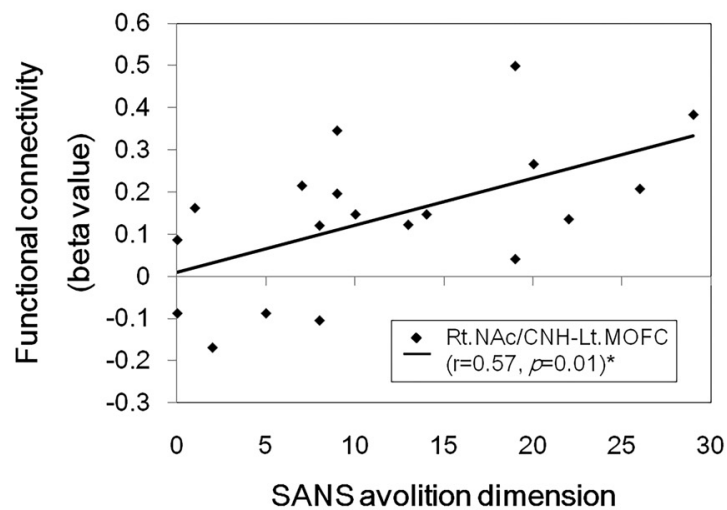

Figure 7. Relationship of the functional connectivity strength of brain regions related to effort and reward-based valuation with the anticipation $(\boldsymbol{A})$ and effort-related reaction time changes ( $\boldsymbol{B}$ ) in healthy controls and the avolition-dimension scores (i.e., the sum of SANS avolition-apathy and anhedonia-associality items) of the SANS in patients with depression (C) and schizophrenia (D). Lt.LOFC, Left lateral orbitofrontal cortex; Lt.MOFC, left medial orbitofrontal cortex; Lt. and Rt.NAc/CHN, right and right NAc/caudate nucleus head; Rt.ACC, right anterior cingulate cortex; Rt.PT, right putamen. Statistically significant at ${ }^{*} p<0.025$ or ${ }^{* *} p<0.0125$.

bance in local circuitry may disrupt reward processing, as suggested by prior evidence demonstrating variability and heterogeneity in cortical activation due to synchronizing failure by disturbed GABAergic neurons (Lewis, 2000).

We also found that positive symptom severity was associated with increasing VTA/SN- NAc/CNH and decreasing putamenmedial OFC connectivity strengths in schizophrenia patients. This corresponds to a prior study showing an association of hallucination with NAc and VTA functional connectivity (Rolland et al., 2015) and provides additional evidence of the hypothesized role of aberrant dopaminergic firing in positive symptoms (Heinz and Schlagenhauf, 2010).

\section{Limitations}

Our findings need to be interpreted with consideration of medication effects on regional and interregional functioning. Prior studies have shown that antidepressants increased sustained NAc and fronto-striatal connectivity while regulating positive affect in depressed patients, whereas reward-learning signals were decreased in the NAc and ACC and increased in the midbrain in treatment-unresponsive depressed patients (Kumar et al., 2008; Heller et al., 2013). However, we did not detect any correlations between antidepressant dosages and striatal-prefrontal functional connectivities during resting state. One possible explanation is that antidepressant effect may lie in indirectly modulating functional connectivity during activated states. Moreover, we observed that stronger NAc/CNH-medial OFC con- nectivity was not associated with improvement in motivation, but instead predicted severity of amotivation. Accordingly, our finding of deficient putamen-medial OFC resting-state connectivity in depressed patients would more likely represent diagnostic specificity than medication effect.

Recent studies have shown that antipsychotics improve striatal-prefrontal and meso-thalamic functional connectivities, which are associated with improvement in psychotic symptoms in schizophrenia (Hadley et al., 2014; Sarpal et al., 2015). Although greater ACC-to-NAc/CNH and putamen functional connectivities were associated with higher antipsychotic dosage in our study, we did not find them to be associated with avolition in schizophrenia patients. However, because the medial OFC is adjacent to ACC, our results suggest that antipsychotic effect in enhancing striatal-prefrontal connectivity may aggravate amotivation. This untoward effect may explain why negative symptoms in schizophrenia are difficult to treat. We speculate that developing treatments targeting improvement in meso-prefrontal connectivity may improve amotivation in schizophrenia.

We analyzed patients who were less educated than healthy controls. However, we did not find significant associations between education and task reaction times, activities, or resting-state functional connectivities except the VTA/SN-right NAc/CNH functional connectivity in patients with depression. This limited effect of education on the VTA/SN functional connectivities in patients with depression insufficiently explains our findings. 


\section{Conclusion}

In conclusion, we propose that difference in striato-orbitofrontal dysfunction in effort-based reinforcement between depression and schizophrenia may be related to difference in the extent of dopaminergic pathway dysfunction. Our findings underscore the need for studies to compare psychiatric disorders with common psychopathology to understand their pathophysiology and to support the role of functional neuroimaging to investigate potential biomarkers for differential diagnosis.

Note Added in Proof: The first author name and affiliation along with second author affiliation was accidentally incorrectly listed, and labels for the graphs in Figure 4 were not included in the Early Release version. The author name, affiliations, and figure have now been corrected.

\section{References}

Andreasen NC (1984a) Scale for the Assessment of Negative Symptoms (SANS). Iowa City: University of Iowa.

Andreasen NC (1984b) Scale for the Assessment of Positive Symptoms (SAPS). Iowa City: University of Iowa.

Assadi SM, Yücel M, Pantelis C (2009) Dopamine modulates neural networks involved in effort-based decision-making. Neurosci Biobehav Rev 33:383-393. CrossRef Medline

Baldwin AE, Sadeghian K, Kelley AE (2002) Appetitive instrumental learning requires coincident activation of NMDA and dopamine D1 receptors within the medial prefrontal cortex. J Neurosci 22:1063-1071. Medline

Beck AT, Ward CH, Mendelson M, Mock J, Erbaugh J (1961) An inventory for measuring depression. Arch Gen Psychiatry 4:561-571. CrossRef Medline

Calhoon GG, O'Donnell P (2013) Closing the gate in the limbic striatum: Prefrontal suppression of hippocampal and thalamic inputs. Neuron 78: 181-190. CrossRef Medline

Cox SM, Frank MJ, Larcher K, Fellows LK, Clark CA, Leyton M, Dagher A (2015) Striatal D1 and D2 signaling differentially predict learning from positive and negative outcomes. Neuroimage 109:95-101. CrossRef Medline

Croxson PL, Walton ME, O’Reilly JX, Behrens TE, Rushworth MF (2009) Effort-based cost-benefit valuation and the human brain. J Neurosci 29: 4531-4541. CrossRef Medline

Deichmann R, Gottfried JA, Hutton C, Turner R (2003) Optimized EPI for fMRI studies of the orbitofrontal cortex. Neuroimage 19:430-441. CrossRef Medline

Dreyer JK, Herrik KF, Berg RW, Hounsgaard JD (2010) Influence of phasic and tonic dopamine release on receptor activation. J Neurosci 30:1427314283. CrossRef Medline

Eshel N, Roiser JP (2010) Reward and punishment processing in depression. Biol Psychiatry 68:118-124. CrossRef Medline

Fellows LK, Farah MJ (2003) Ventromedial frontal cortex mediates affective shifting in humans: evidence from a reversal learning paradigm. Brain 126:1830-1837. CrossRef Medline

Foussias G, Remington G (2010) Negative symptoms in schizophrenia: avolition and Occam's razor. Schizophr Bull 36:359-369. CrossRef Medline

Gläscher J, Hampton AN, O'Doherty JP (2009) Determining a role for ventromedial prefrontal cortex in encoding action-based value signals during reward-related decision making. Cereb Cortex 19:483-495. CrossRef Medline

Gold JM, Strauss GP, Waltz JA, Robinson BM, Brown JK, Frank MJ (2013) Negative symptoms of schizophrenia are associated with abnormal effortcost computations. Biol Psychiatry 74:130-136. CrossRef Medline

Grace AA, Floresco SB, Goto Y, Lodge DJ (2007) Regulation of firing of dopaminergic neurons and control of goal-directed behaviors. Trends Neurosci 30:220-227. CrossRef Medline

Grieder TE, George O, Tan H, George SR, Le Foll B, Laviolette SR, van der Kooy D (2012) Phasic D1 and tonic D2 dopamine receptor signaling double dissociate the motivational effects of acute nicotine and chronic nicotine withdrawal. Proc Natl Acad Sci U S A 109:3101-3106. CrossRef Medline

Hadley JA, Nenert R, Kraguljac NV, Bolding MS, White DM, Skidmore FM, Visscher KM, Lahti AC (2014) Ventral tegmental area/midbrain functional connectivity and response to antipsychotic medication in schizophrenia. Neuropsychopharmacology 39:1020-1030. CrossRef Medline
Hamilton M (1960) A rating scale for depression. J Neurol Neurosurg Psychiatry 23:56-62. CrossRef Medline

Heinz A, Schlagenhauf F (2010) Dopaminergic dysfunction in schizophrenia: salience attribution revisited. Schizophr Bull 36:472-485. CrossRef Medline

Heller AS, Johnstone T, Light SN, Peterson MJ, Kolden GG, Kalin NH, Davidson RJ (2013) Relationships between changes in sustained fronto-striatal connectivity and positive affect in major depression resulting from antidepressant treatment. Am J Psychiatry 170:197206. CrossRef Medline

Hurd YL, Suzuki M, Sedvall GC (2001) D1 and D2 dopamine receptor mRNA expression in whole hemisphere sections of the human brain. J Chem Neuroanat 22:127-137. CrossRef Medline

Knutson B, Wimmer GE (2007) Splitting the difference: how does the brain code reward episode? Ann N Y Acad Sci 1104:54-69. CrossRef Medline

Knutson B, Bhanji JP, Cooney RE, Atlas LY, Gotlib IH (2008) Neural responses to monetary incentives in major depression. Biol Psychiatry 63: 686-692. CrossRef Medline

Kravitz AV, Tye LD, Kreitzer AC (2012) Distinct roles for direct and indirect pathway striatal neurons in reinforcement. Nat Neurosci 15:816-818. CrossRef Medline

Kumar P, Waiter G, Ahearn T, Milders M, Reid I, Steele JD (2008) Abnoraml temporal difference reward-learning signals in major depression. Brain 131:2084-2093. CrossRef Medline

Kurniawan IT, Seymour B, Talmi D, Yoshida W, Chater N, Dolan RJ (2010) Choosing to make an effort: The role of striatum in signaling physical effort of a chosen action. J Neurophysiol 104:313-321. CrossRef Medline

Lee JS, Chun JW, Kang JI, Kang DI, Park HJ, Kim JJ (2012) Hippocampus and nucleus accumbens activity during neutral word recognition related to trait physical anhedonia in patients with schizophrenia: an fMRI study. Psychiatry Res 203:46-53. CrossRef Medline

Lee JS, Jung S, Park IH, Kim JJ (2015) Neural basis of anhedonia and amotivation in patients with schizophrenia: the role of reward system. Curr Neuropharmacol 13:750-759. CrossRef Medline

Lee YM, Park IH, Koo MS, Ko SY, Kang HM, Song JE (2013) The reliability and validity of the Korean version of Apathy Evaluation Scale and its application in patients with schizophrenia. Korean Journal of Schizophrenia Research 16:80-85. CrossRef

Lewis DA (2000) GABAergic local circuit neurons and prefrontal cortical dysfunction in schizophrenia. Brain Res Rev 31:270-276. CrossRef Medline

Marin RS, Biedrzycki RC, Firinciogullari S (1991) Reliability and validity of the apathy evaluation scale. Psychiatry Res 38:143-162. CrossRef Medline

Murray GK, Corlett PR, Clark L, Pessiglione M, Blackwell AD, Honey G, Jones PB, Bullmore ET, Robbins TW, Fletcher PC (2008) Substantia nigra/ventral tegmental reward prediction error disruption in psychosis. Mol Psychiatry 13:239, 267-276. CrossRef Medline

Nielsen MØ, Rostrup E, Wulff S, Bak N, Lublin H, Kapur S, Glenthøj B (2012) Alterations of the brain reward system in antipsychotic naïve schizophrenia patients. Biol Psychiatry 71:898-905. CrossRef Medline

O’Doherty J, Dayan P, Schultz J, Deichmann R, Friston K, Dolan RJ (2004) Dissociable roles of ventral and dorsal striatum in instrumental conditioning. Science 304:452-454. CrossRef Medline

Park IH, Chun JW, Park HJ, Koo MS, Park S, Kim SH, Kim JJ (2015) Altered cingulo-striatal function underlies reward drive deficits in schizophrenia. Schizophr Res 161:229-236. CrossRef Medline

Pizzagalli DA, Holmes AJ, Dillon DG, Goetz EL, Birk JL, Bogdan R, Dougherty DD, Iosifescu DV, Rauch SL, Fava M (2009) Reduced caudate and nucleus accumbens response to rewards in unmedicated individuals with major depressive disorder. Am J Psychiatry 166:702-710. CrossRef Medline

Rolland B, Amad A, Poulet E, Bordet R, Vignaud A, Bation R, Delmaire C, Thomas P, Cottencin O, Jardri R (2015) Resting-state functional connectivity of the nucleus accumbens in auditory and visual hallucinations in schizophrenia. Schizophr Bull 41:291-299. CrossRef Medline

Sarpal DK, Robinson DG, Lencz T, Argyelan M, Ikuta T, Karlsgodt K, Gallego JA, Kane JM, Szeszko PR, Malhotra AK (2015) Antipsychotic treatment and functional connectivity of the striatum in first-episode schizophrenia. JAMA Psychiatry 72:5-13. CrossRef Medline

Simon JJ, Biller A, Walther S, Roesch-Ely D, Stippich C, Weisbrod M, Kaiser S (2010) Neural correlates of reward processing in schizophrenia: rela- 
tionship to apathy and depression. Schizophr Res 118:154-161. CrossRef Medline

Strauss GP, Frank MJ, Waltz JA, Kasanova Z, Herbener ES, Gold JM (2011) Deficits in positive reinforcement learning and uncertainty-driven exploration are associated with distinct aspects of negative symptoms in schizophrenia. Biol Psychiatry 69:424-431. CrossRef Medline

Treadway MT, Bossaller NA, Shelton RC, Zald DH (2012a) Effort-based decisionmaking in major depressive disorder: A translational model of motivational anhedonia. J Abnorm Psychol 121:553-558. CrossRef Medline

Treadway MT, Buckholtz JW, Cowan RL, Woodward ND, Li R, Ansari MS, Baldwin RM, Schwartzman AN, Kessler RM, Zald DH (2012b) Dopaminergic mechanisms of individual differences in human effort-based decision-making. J Neurosci 32:6170-6176. CrossRef Medline
Tzourio-Mazoyer N, Landeau B, Papathanassiou D, Crivello F, Etard O, Delcroix N, Mazoyer B, Joliot M (2002) Automated anatomical labeling of activations in SPM using a macroscopic anatomical parcellation of the MNI MRI single-subject brain. Neuroimage 15:273-289. CrossRef Medline

Waltz JA, Frank MJ, Robinson BM, Gold JM (2007) Selective reinforcement learning deficits in schizophrenia support predictions from computational models of striatal-cortical dysfunction. Biol Psychiatry 62:756-764. CrossRef Medline

Waltz JA, Schweitzer JB, Ross TJ, Kurup PK, Salmeron BJ, Rose EJ, Gold JM, Stein EA (2010) Abnormal responses to monetary outcomes in cortex, but not in the basal ganglia, in schizophrenia. Neuropsychopharmacology 35:2427-2439. CrossRef Medline 